\title{
Infinite dimensional stochastic differential equations and their applications
}

\author{
By
}

\author{
Tokuzo SHIGA and Akinobu SHIMIZU
}

(Communicated by Prof. S. Watanabe, March 27, 1979)

\section{§ 1. Introduction}

The central aim of this paper is to construct some classes of infinite dimensional stochastic processes related to population genetics or statistical mechanics by making use of infinite dimensional stochastic differential equations.

To begin with, we will explain an example related to population genetics. Let $S$ be a countable set which we consider as the set of colonies. Suppose that there are two alleles $A$ and $a$ in each colony and that the change of gene frequencies is caused by random sampling, mutation, selection and migration. As is well-known in population genetics ([1], [6]), when we ignore the migration effect, the frequency $x_{i}(t)$ of $A$-genes in the $i$-th colony at time $t$ may be considered a path of 1 -dimensional diffusion process on the interval $[0,1]$ determined by the diffusion coefficient

$$
-\frac{1}{4 N} x_{i}\left(1-x_{i}\right)
$$

and the drift coefficient

$$
v-(u+v) x_{i}+s x_{i}\left(1-x_{i}\right)
$$

for each $i \in S$. Hence the frequency $x_{i}(t)$ satisfies the following 1-dimensional stochastic differential equation

$$
d x_{i}(t)=\sqrt{\frac{1}{2 N} x_{i}\left(1-x_{i}\right) d B_{i}(t)+\left(v-(u+v) x_{i}+s x_{i}\left(1-x_{i}\right)\right) d t}
$$

for each $i \in S$, where $\left\{B_{i}(t)\right\}_{i \in S}$ is an independent system of 1-dimens- 
ional Brownian motions. Now, we suppose that $q_{j i}$ stands for the rate of migration from the $j$-th colony to the $i$-th. It seems quite natural to consider the following stochastic differential equation

$$
\begin{aligned}
d x_{i}(t) & =\sqrt{\frac{1}{2 N} x_{i}\left(1-x_{i}\right)} d B_{i}(t)+\left(v-(u+v) x_{i}+s x_{i}\left(1-x_{i}\right)\right. \\
& \left.+\sum_{j \in S} q_{j i} x_{j}\right) d t, \quad i \in S .
\end{aligned}
$$

Such a system should be called a continuous time stepping stone model. The discrete time stepping stone model was proposed by $M$. Kimura for the problem of local differentiation of gene frequencies and it has been studied by many biologists. (cf. J. F. Crow \& M. Kimura [1]). Naturally, we expect that $E q .(1.1)$ has a unique solution $X(t)=\left\{x_{i}(t)\right\}_{i \in s}$ taking values in the space $[0,1]^{s}$. In $\S 3$, we shall discuss the following stochastic differential equation, which is more general than $E q .(1.1)$,

$$
d x_{i}(t)=\alpha_{i}\left(x_{i}(t)\right) d B_{i}(t)+f_{i}(X(t)) d t, \quad i \in S,
$$

where $X(t)=\left\{x_{i}(t)\right\}_{i \in s}$, and $\left\{B_{i}(t)\right\}_{i \in s}$ is an independent system of 1dimensional Brownian motions and we shall give a sufficient condition under which $E q .(1.2)$ has a unique strong solution $X(t)=\left\{x_{i}(t)\right\}_{i \in S} \in$ $[0,1]^{s}$ for any initial data $X(0) \in[0,1]^{s}$. Ergodic behaviors of the system (1.1) have been discussed by one of the authors [11].

Secondly, we suppose that there are infinite many alleles $A_{1}, A_{2}, \ldots$, $A_{n}, \ldots$ in each colony. We denote by $x_{i}^{n}(t)$ the frequency of $A_{n}$-genes in the $i$-th colony at time $t$. Then $x_{i}^{n}(t)$ satisfies the stochastic differential equation (1.3) under some genetical assumptions;

$$
\begin{gathered}
d x_{i}^{n}(t)=\sum_{m=1}^{n} \alpha_{i}^{n m}\left(x_{i}^{1}, x_{i}^{2}, \ldots, x_{i}^{n}\right) d B_{i}^{m}+\left(\mu\left(x_{i}^{n-1}-x_{i}^{n}\right)+\sum_{j \in S} q_{j i} x_{j}^{n}\right) d t \\
x_{i}^{0}(t)=0, \quad i \in S, n \in N
\end{gathered}
$$

where $N$ is the set of natural numbers, the functions $\alpha_{i}^{n m}, i \in S, n, m \in N$ are continuous on the $n$-dimensional set $\left\{\left(x^{1}, x^{2}, \ldots, x^{n}\right) ; x^{k} \geqq 0\right.$ for each $k$ and $\left.\sum_{k=1}^{n} x^{k} \leqq 1\right\}$, the triangular matrix $A=\left(\alpha_{i}^{n m}\right)_{n \geq m}$ is determined by the relation

$$
A A^{t}=\left(\sigma^{2} x^{n}\left(\delta_{n, m}-x^{m}\right)\right)_{n, m \in N}, \text { where } \sigma^{2} \geqq 0 \text { and } \delta_{n, m}= \begin{cases}1 & (n=m) \\ 0 & (n \neq m)\end{cases}
$$

and $\left\{B_{i}(t)\right\}_{i \in S}=\left\{\left(B_{i}^{1}, B_{i}^{2}, \ldots, B_{i}^{n}, \ldots\right)\right\}_{i \in S}$ is an independent system of infinite dimesional Brownian motions. 
We shall prove in $\S 3$ that $E q .(1.3)$ has a unique strong solution $X(t)=\left\{x_{i}^{n}(t)\right\}_{n \in N, i \in S} \in\left(L_{\infty}\right)^{s}$ for any initial data $X(0) \in\left(L_{\infty}\right)^{s}$ where $L_{\infty}=$ $\left\{X=\left(x^{1}, x^{2}, \ldots, x^{n}, \ldots\right) ; x^{n} \geqq 0\right.$ for any $n \in N$ and $\left.\sum_{n \in N} x^{n}=1\right\}$.

Finally, in $\S 4$ we shall discuss diffusion processes on $\mathscr{S}^{\prime}\left(Z^{d}\right)$, the space of all tempered sequences on $Z^{d}$. It enable us to construct a time evolution of an unbounded spin system in statistical mechanics as a diffusion process on $\mathscr{S}^{\prime}\left(Z^{d}\right)$ determined by the following stochastic differential equation

$$
d x_{i}(t)=d B_{i}(t)-\frac{1}{2}\left(\Phi_{i}^{\prime}\left(x_{i}(t)\right)+\sum_{j+i} \Phi_{i, j}^{\prime}\left(x_{i}(t), x_{j}(t)\right)\right) d t,
$$

where $B(t)=\left\{B_{i}(t)\right\}_{i \in z^{d}}$ is an independent system of 1-dimensional Brownian motions, $\Phi_{i}(x), i \in Z^{d}$ are self-potential functions, $\Phi_{i, j}(x, y), i, j \in Z^{d}$ are pair potential functions and $\Phi_{i, j}^{\prime}(x, y)=\frac{\partial}{\partial x} \Phi_{i, j}(x, y)$.

Recently H. Doss and G. Royer [2] constructed a diffusion process associated with (1.4). However, our method which is based on a standard approximation procedure in the theory of stochastc differential equations, seems to be more natural.

\section{§ 2. Preliminaries}

Let $S$ be a countable set. Let, for each $i \in S, \alpha_{i}$ be a continuous mapping from $d$-dimensional Euclidean space $R^{d}$ into the space of $d \times d$ matrices $R^{d} \otimes R^{d}$ and $f_{i}$ be a mapping from $\left(R^{d}\right)^{s}$ into $R^{d}$ which is continuous in the product topology of $\left(R^{d}\right)^{S}$. Each point of the space $\left(R^{d}\right)^{S}$ is denoted by $X=\left\{x_{i}\right\}_{i \in S}$ where $x_{i} \in R^{d}$. $\left|x_{i}\right|$ denotes the $d$-dimensional Euclidean norm of $x_{i}$ and $\left|\alpha_{i}\right|^{2}=\sum_{n, m=1}^{d}\left(\alpha_{i}^{n m}\right)^{2}$, where $\alpha_{i}^{n m}$ is the $(n, m)$-component of $\alpha_{i}$.

In this section, we shall discuss the following stochastic differential equation;

$$
x_{i}(t)=x_{i}(0)+\int_{0}^{t} \alpha_{i}\left(x_{i}(s)\right) d B_{i}(s)+\int_{0}^{t} f_{i}(X(s)) d s, \quad i \in S,
$$

where $B(t)=\left\{B_{i}(t)\right\}_{i \in s}$ is an independent system of $d$-dimensional Brownian motions. We will give the definition of a solution of Eq.(2.1).

Definition. A pair $(X, B)=(X(t), B(t))_{t \geq_{0}}$ defined on a probability space $\left(\Omega, \mathscr{F}, P ; \mathscr{F}_{l}\right)$ is called a solution of $(2.1)$ if the following conditions (i) - (iii) are satisfied;

(i) $B(t)=\left\{B_{i}(t)\right\}_{i \in S}$ is an independent system of $d$-dimensional $\mathscr{F}_{t^{-}}$ 
Brownian motions with $B(0)=0$,

(ii) $X(t)=\left\{x_{i}(t)\right\}_{i \in s}$ is a $\mathscr{F}_{i}$-adapted stochastic process and $x_{i}(t)$ is continuous in $t \geqq 0$ with probability one for each $i \in S$ and

(iii) $(X(t), B(t))_{t \geq 0}$ satisfies the stochastic differential equation (2.1) with probability one.

First, we shall discuss the existence of a solution of $E q .(2.1)$ under the following assumption.

Let $\gamma=\left\{\gamma_{i}\right\}_{i \in S}$ be a positive sequence on $S$ and let $L^{2}(\gamma)$ be the space of all square $\gamma$-summable sequences on $S$, i. e.

$$
L^{2}(\gamma)=\left\{X \in\left(R^{d}\right)^{s} ;\|X\|_{r}^{2}=\sum_{i \in S} \gamma_{i}\left|x_{i}\right|^{2}<+\infty\right\} .
$$

Assumption $[A]$ For some positive constant $C$, it holds that

$$
\sum_{i \in S} \gamma_{i}\left|\alpha_{i}\left(x_{i}\right)\right|^{2} \leqq C\left(1+\|X\|_{r}^{2}\right)
$$

and

$$
\sum_{i \in S} \gamma_{i}\left|f_{i}(X)\right|^{2} \leqq C\left(1+\|X\|_{r}^{2}\right) \text { for any } X \in L^{2}(\gamma) .
$$

Then we get

Theorem 2. 1. Let $X \in L^{2}(\gamma)$. Then there exists a solution $(X(t), B(t))$ of Eq.(2.1) on a probability space $\left(\Omega, \mathscr{F}, P ; \mathscr{F}_{t}\right)$ such that

$$
P[X(0)=X]=1
$$

and

(2.5) $P\left[X(t)\right.$ is a $L^{2}(\gamma)$-valued strongly continuous function of $\left.t \geqq 0\right]=1$.

Proof. Let $\left\{S_{n}\right\}$ be a sequence of finite subsets of $S$ such that $S_{n} \nearrow S$ as $n \rightarrow \infty$. Let us consider the following equation;

$$
\begin{aligned}
& x_{i}(t)=x_{i} \quad i \notin S_{n} \\
& x_{i}(t)=x_{i}+\int_{0}^{t} \alpha_{i}\left(x_{i}^{n}(s)\right) d B_{i}^{n}(s)+\int_{0}^{t} f_{i}\left(X^{n}(s)\right) d s, \quad i \in S_{n} .
\end{aligned}
$$

By Skorohod's existence theorem of finite dimensional case [12], there exists a solution $\left(X^{n}(t), B^{n}(t)\right)=\left\{\left(x_{\boldsymbol{i}}^{n}(t), B_{i}^{n}(t)\right)\right\}_{i \in s}$ on a probability space $\left(\Omega^{n}, \mathscr{F}^{n}, P^{n} ; \mathscr{F}_{t}^{n}\right)$ for each $n$.

Then we can easily see that for each finite $T$ there exists a constant $C^{\prime}>0$ such that 


$$
\sup _{n} E^{n}\left[\sup _{0 \leqq t \leqq T}\left\|X^{n}(t)\right\|_{r}^{2}\right]<+\infty
$$

and

$$
\sup _{n} E^{n}\left[\left\|X^{n}(t)-X^{n}(s)\right\|_{r}^{2}\right] \leqq C^{\prime}|t-s|, \quad \text { for } 0 \leqq t, s \leqq T .
$$

Thus, we see

$$
\begin{gathered}
\sup _{n} P^{n}\left[\sup _{0 \leq t \leq T}\left|x_{i}^{n}(t)\right|>C\right] \rightarrow 0, \text { as } C \rightarrow \infty \text {, and } \\
\sup _{n} \sup _{\substack{t, t \leq \mid \leq h \\
0 \leq t, s \leq T}} P^{n}\left[\left|x_{i}^{n}(t)-x_{i}^{n}(s)\right|>\varepsilon\right] \rightarrow 0, \text { as } h \rightarrow 0, \quad i \in S
\end{gathered}
$$

for any $\varepsilon>0$. By making use of Skorohod's argument, we can see that there exist a sequence $\left\{n_{p}\right\}$, a sequence of stochastic processes $\left(\tilde{X}^{n} p(t), \tilde{B}^{n} p(t)\right)$ and a stochastic process $(\tilde{X}(t), \tilde{B}(t))$ defined on a probability space $(\tilde{\Omega}$, $\tilde{\mathscr{F}}, \tilde{P})$ such that finite dimesional distributions of $\left(\tilde{X}^{n} p(t), \tilde{B}^{n}(t)\right)$ and $\left(X^{n} p(t), B^{n} p(t)\right)$ coincide and $\left(\tilde{x}_{i}^{n} p(t), \tilde{B}_{i}^{n_{p}}(t)\right)$ converges to $\left(\tilde{x}_{i}(t), \tilde{B}_{i}(t)\right)$ in probability as $p \rightarrow+\infty$ for any $t$ and $i \in S$. We may assume that the processes $\tilde{x}_{i}^{n}{ }_{p}(t)$ have continuous trajectories and that $\tilde{B}^{n}{ }^{p}(t)$ and $\tilde{B}(t)$ are independent systems of $d$-dimensional Brownian motions.

It is known (cf. N. V. Krylov [4]) that the pair $\left(\tilde{X}^{n} p(t), \tilde{B}^{n} p(t)\right.$ ) satisfies the finite dimensional stochastic differential equation

$$
\begin{aligned}
& \tilde{x}_{i}^{n}{ }^{p}(t)=x_{i}, \quad i \notin S_{n_{p}} \\
& \tilde{x}_{i}^{n}{ }_{p}(t)=x_{i}+\int_{0}^{t} \alpha_{i}\left(\tilde{x}_{i}^{n}{ }_{p}^{p}(s)\right) d \tilde{B}_{i}^{n}(t)+\int_{0}^{t} f_{i}\left(\tilde{X}^{n} p(s)\right) d s, \quad i \in S_{n_{p}} .
\end{aligned}
$$

Since $\alpha_{i}\left(\tilde{x}_{i}^{n}{ }^{p}(t)\right)$ converges to $\alpha_{i}\left(\tilde{x}_{i}(t)\right)$ in probability, we see that $\int_{0}^{t} \alpha_{i}$ $\left(\tilde{x}_{i}^{n}{ }^{p}(s)\right) d \tilde{B}_{i}^{n}{ }^{p}(s)$ converges to $\int_{0}^{t} \alpha_{i}\left(\tilde{x}_{i}(s)\right) d \tilde{B}_{i}(s)$ in probability as $p \rightarrow+\infty$. (cf. A. V. Skorohod [12], p. 32).

On the other hand, we can see that $\left\{f^{n_{p}}\left(\tilde{X}^{n_{p}}(t, \tilde{\omega})\right)\right\}_{p \geqq 1}$ is uniformly integrable with respect to the measure $d t \times \tilde{P}(d \tilde{\omega})$ on $[0, T] \times \tilde{\Omega}$ because

$$
\sup _{p} \int_{0}^{T} d t \tilde{E}\left[\left|f_{i}\left(X^{n} p(t, \tilde{\omega})\right)\right|^{2}\right]<+\infty .
$$

Noting that $\tilde{X}^{n} p(t, \tilde{\omega})$ converges to $\tilde{X}(t, \tilde{\omega})$ in measure with respect to $d t \times \tilde{P}(d \tilde{\omega})$ on $[0, T] \times \tilde{\Omega}(T<+\infty)$, we see that there exists a subsequence $\left\{n_{p}^{\prime}\right\}$ of $\left\{n_{p}\right\}$ such that $\tilde{X}^{n}{ }^{\prime}(t, \tilde{\omega})$ converges to $\tilde{X}(t, \tilde{\omega})$ almost everywhere $(t, \tilde{\omega})$ with respect to $d t \times \tilde{P}(d \tilde{\omega})$. Taking into consideration of the continuity of $f_{i}(X)$, we have 


$$
\int_{0}^{T} d t \tilde{E}\left[\left|f_{i}\left(\tilde{X}^{n^{\prime}}(t, \tilde{\omega})\right)-f_{i}(\tilde{X}(t, \tilde{\omega}))\right|\right] \rightarrow 0 \text { as } p \rightarrow+\infty \text {. }
$$

Thus, we see that the pair $(\tilde{X}(t), \tilde{B}(t))_{t \geq 0}$ satisfies

$$
\tilde{x}_{i}(t)-x_{i}=\int_{0}^{t} \alpha_{i}\left(\tilde{x}_{i}(s)\right) d \tilde{B}_{i}(s)+\int_{0}^{t} f_{i}(\tilde{X}(s)) d s, \quad i \in S, \text { a.s. }
$$

for each $t$. The right hand side of this equality has a continuous modification for each $i \in S$ which we denote by $\hat{x}_{i}(t)-x_{i}$. Put

$$
\mathscr{F}_{t}=\bigcap_{c>0}\left[\sigma\{\hat{X}(s), \tilde{B}(s), s \leqq t+\varepsilon\}^{\vee}, \mathcal{N}\right]
$$

where $\mathscr{N}$ stands for all subsets of $\tilde{\Omega}$ with $\tilde{P}$-measure 0 . Then, it is clear that $(\hat{X}(t), \tilde{B}(t))_{t \geq 0}$ is a solution defined on $\left(\tilde{\Omega}, \mathscr{F}, \tilde{P} ; \mathscr{F}_{t}\right)$ of Eq. (2. 1)

Finally we note that $\hat{X}(t)$ is a $L^{2}(\gamma)$-valued strongly continuous function of $t \geqq 0$ with probability one. To show this it is sufficient to see that

$$
\tilde{P}\left[\sum_{i \in S} \gamma_{i} \sup _{0 \leq t \leq T}\left|\int_{0}^{t} \alpha_{i}\left(x_{i}(s)\right) d B_{i}(s)\right|^{2}<+\infty \text { for any } T<\infty\right]=1
$$

and

$$
\tilde{P}\left[\sum_{i \in S} \gamma_{i}\left(\int_{0}^{T}\left|f_{i}(X(s))\right| d s\right)^{2}<+\infty \text { for any } T<\infty\right]=1 .
$$

It follows from (2.7) that

$$
\sup _{0 \leqq t \leq T} \tilde{E}\left[\|\hat{X}(t)\|_{r}^{2}\right]<+\infty \text { for any finite } T .
$$

By using (2. 14) and a maximal inequality for martingales, we can sce

(2. 15) $\sum_{i \in S} \gamma_{i} \tilde{E}\left[\sup _{0 \leq t \leq T}\left|\int_{0}^{t} \alpha_{i}\left(\hat{x}_{i}(s)\right) d \tilde{B}_{i}(s)\right|^{2}\right]<+\infty$ for any finite $T$.

Thus (2.12) is valid. Also, (2. 13) follows immediately from (2. 14).

Remark 2.1. Suppose that for some constant $C>0 \quad\left|\alpha_{i}\left(x_{i}\right)\right| \leqq C$ and $\left|f_{i}(X)\right| \leqq C$ hold any $x_{i} \in R^{d}$, any $X \in\left(R^{d}\right)^{s}$ and any $i \in S$. Then, Assumption $[A]$ holds for any positive sequence $\gamma=\left\{\gamma_{i}\right\}$ with $\sum_{i} \gamma_{i}<+\infty$. Moreover, if $\sup _{i}\left|x_{i}\right|<+\infty$, there exists a solution of $E q(2.1)$ such that $\sup _{i} E$ $\left[\sup _{0 \leq t \leq T}\left|x_{i}(t)\right|^{2}\right]<+\infty$.

Let $K$ be a compact set of $R^{d}$. A solution $(X(t), B(t))_{t \geq 0}$ of Eq.(2.1) defined on $\left(\Omega, \mathscr{F}, P ; \mathscr{F}_{t}\right)$ is called $L^{2}(\gamma)$-valued $\left(K^{s}\right.$-valued) if $X(t) \in$ $L^{2}(\gamma)$ (resp. $X(t) \in K^{s}$ ) for all $t \geqq 0$ almost surely. Theorem 2.1 asserts the existence of a $L^{2}(\gamma)$-valued solution under Assumption [A], and it will 
be discussed in $\S 3$ the case that $E q .(2.1)$ has a $K^{s}$-valued solution. We will omit the definitions of the pathwise uniqueness of $L^{2}(\gamma)$-valued (or $K^{s}$-valued) solutions and of a $L^{2}(\gamma)$-valued (or $K^{s}$-valued) strong solution because these definitions can be given in the same way as in the theory of finite dimensional stochastic differential equations. (cf. T. Yamada \& S. Watanabe [13])

Thus, we obtain

Theorem 2. 2. The existence of a $L^{2}(\gamma)$-valued ( $K^{s}$-valued) solution and the pathwise uniqueness of $L^{2}(\gamma)$-valued ( $K^{s}$-valued) solutions imply the existence of a unique $L^{2}(\gamma)$-valued (resp. $K^{s}$-valued) strong solution.

\section{§ 3. Stochastic differential equations related to stepping stone models}

First, we consider the following stochastic differential equation

(3. 1) $d x_{i}^{n}(t)=\sum_{m=1}^{d} \alpha_{i}^{n m}\left(x_{i}(t)\right) d B_{i}^{m}(t)+f_{i}^{n}(X(t)) d t \quad i \in S, 1 \leqq n \leqq d$,

where $B(t)=\left\{\left(B_{i}^{1}(t), B_{i}^{2}(t), \ldots, B_{i}^{d}(t)\right\}_{i \in S}\right.$ is an independent system of $d$-dimensional Brownian motions. We denote by $L$ the $d$-dimensional set $\left\{x=\left(x^{1}, x^{2}, \ldots, x^{d}\right) \in R^{d} ; x^{k} \geqq 0\right.$ for each $k$ and $\left.\sum_{k=1}^{d} x^{k} \leqq 1\right\}$.

Let us consider Eq.(3.1) under the following assumptions.

Assumption [B-1] $\alpha_{i}^{n m}(x), i \in S, 1 \leqq n, m \leqq d$ are continuous functions defined on the set $L$, uniformly bounded in $i \in S$ and $1 \leqq n, m \leqq d$, and satisfy the following conditions; for each $i \in S$,

$$
\begin{aligned}
& {\left[\alpha_{i} \cdot \alpha_{i}^{t}\right]^{n n}(x)=0 \quad \text { if } x=\left(x^{1}, \ldots, x^{d}\right) \in L \text { and } x^{n}=0, \text { and }} \\
& \sum_{n=1}^{d} \sum_{m=1}^{d}\left[\alpha_{i} \cdot \alpha_{i}^{t}\right]^{n m}(x)=0 \quad \text { if } x=\left(x^{1}, \ldots, x^{d}\right) \in L \text { and } \sum_{n=1}^{d} x^{n}=1 .
\end{aligned}
$$

Assumption [B-2] $f_{i}^{n}(X), \quad i \in S, 1 \leqq n \leqq d$ are continuous functions defined on $L^{s}$, equipped with the product topology, such that

$$
\begin{gathered}
\sup _{i \in S} \sup _{x \in L^{S}}\left|f_{i}(X)\right|<+\infty, \\
f_{i}^{n}(X) \geqq 0 \text { if } X=\left\{x_{j}^{m}\right\} \in L^{s} \text { and } x_{i}^{n}=0 \quad i \in S, 1 \leqq n \leqq d,
\end{gathered}
$$

and

$$
\sum_{m=1}^{d} f_{i}^{m}(X) \leqq 0 \quad \text { if } X=\left\{x_{j}^{m}\right\} \in L^{s} \text { and } \sum_{m=1}^{d} x_{i}^{m}=1
$$


Then under Assumptions $[B-1]$ and $[B-2]$ we obtain

Theorem 3. 1. For any $X \in L^{s}$, the stochastic differential equation (3.1) has a solution $(X(t), B(t))_{t \geq 0}$ on a probability space $\left(\Omega, \mathscr{F}, P ; \mathscr{F}_{t}\right)$ such that

$$
P\left[X(0)=X \text { and } X(t) \in L^{s} \text { for all } t \geqq 0\right]=1 \text {. }
$$

Proof. Let $\rho$ be the projection mapping from $R^{d}$ onto $L$, i. e. for each $x, \rho x$ is uniquely determined by $|x-\rho x|=\inf \{|x-y| ; y \in L\}$. Set $\bar{\alpha}_{i}(x)=$ $\alpha_{i}(\rho x)$ and $\bar{f}_{i}(X)=f_{i}(\rho X)$, where $\rho X=\left\{\rho x_{i}\right\}_{i \in S}$ for $X=\left\{x_{i}\right\}_{i \in S} \in\left(R^{d}\right)^{s}$. Then by Remark 2.1 there exists a solution $(X(t), B(t))_{t \geq 0}$ on a probability space $\left(\Omega, \mathscr{F}, P ; \mathscr{F}_{t}\right)$ of the following stochastic differential equation,

$$
\begin{aligned}
& d x_{i}^{n}(t)=\sum_{m=1}^{d} \bar{\alpha}_{i}^{n m}\left(x_{i}(t)\right) d B_{i}^{m}(t)+\bar{f}_{i}^{n}(X(t)) d t \\
& X(0)=X \in L^{s}
\end{aligned}
$$

and it holds that

$$
\sup _{i \in S} E\left[\sup _{0 \leq t \leq T}\left|x_{i}(t)\right|^{2}\right]<+\infty \text { for each finite } T .
$$

To complete this theorem it suffices to show

$$
P\left[X(t) \in L^{s} \text { for all } t \geqq 0\right]=1 \text {. }
$$

For any $\varepsilon>0$, set $u_{\bullet}(y)=\frac{1}{y+\varepsilon} \quad\left(y \in R^{1}\right)$. Then by using Assumptions [B-1] and $[B-2]$, we see easily that there exists a positive constant $K$ such that

$$
\frac{1}{2} u_{c}^{\prime \prime}\left(x_{i}^{n}\right) \sum_{m=1}^{d}\left(\bar{\alpha}_{i}^{n ! m}\left(x_{i}\right)\right)^{2}+u_{c}^{\prime}\left(x_{i}^{n}\right) \bar{f}_{i}^{n}(X) \leqq K \quad \text { if } x_{i}^{n}>-\varepsilon,
$$

and

$$
\begin{aligned}
& \frac{1}{2} u_{\varepsilon}^{\prime \prime}\left(1-\sum_{n=1}^{d} x_{i}^{n}\right) \sum_{n=1}^{d} \sum_{m=1}^{d} \sum_{k=1}^{d} \bar{\alpha}_{i}^{n k} \bar{\alpha}_{i}^{m k}\left(x_{i}\right) \\
& -u_{\varepsilon}^{\prime}\left(1-\sum_{n=1}^{d} x_{i}^{n}\right)\left(\sum_{n=1}^{d} \bar{f}_{i}^{n}(X)\right) \leqq K \quad \text { if } \quad \sum_{n=1}^{d} x_{i}^{n}<1+\varepsilon .
\end{aligned}
$$

Applying Ito's formula we have

$$
E\left[u_{\bullet}\left(x_{i}^{n}\left(t_{\wedge} \tau_{i, n}^{\delta}\right)\right)\right] \leqq u_{\bullet}\left(x_{i}^{n}\right)+K t \quad \text { for any } 0<\delta<\varepsilon
$$

where $\tau_{i, n}^{\delta}$ is defined by $\tau_{i, n}^{\delta}=\inf \left\{t \geqq 0 ; x_{i}^{n}(t) \leqq-\delta\right\}$ for each $i, n$ and $\delta$. So, letting $\delta$ tend to $\varepsilon$, we have $P\left[\tau_{i, n}^{\varepsilon} \leqq t\right]=0$ for any finite $t$ and $\varepsilon>0$. Hence, $P\left[x_{i}^{n}(t) \geqq 0\right.$ for all $\left.t \geqq 0\right]=1$ holds.

In the same way, setting $\tau_{i}^{*}=\inf \left\{t \geqq 0 ; 1-\sum_{n=1}^{d} x_{i}^{n}(t) \leqq-\varepsilon\right\}$, we obtain 


$$
E\left[u_{c}\left(1-\sum_{n=1}^{d} x_{i}^{n}\left(t_{\wedge} \tau_{i}^{c}\right)\right]<+\infty \text { for any finite } t>0 .\right.
$$

Thus, we have $P\left[\sum_{n=1}^{d} x_{i}^{n}(t) \leqq 1\right.$ for all $\left.t \geqq 0\right]=1$ for any $i \in S$.

Remark 3. 1. The following examples are found in population genetics, which satisfy Assumption [B-1].

$$
\begin{aligned}
{\left[\alpha_{i} \cdot \alpha_{i}^{t}\right]^{n m}(x) } & =\sigma^{2} x^{n}\left(\delta_{n, m}-x^{m}\right) \\
{\left[\alpha_{i} \cdot \alpha_{i}^{t}\right]^{n m}(x) } & =\sigma^{2} x^{n}\left(\delta_{n, m}-x^{m}\right)+x^{n} x^{m}\left(V_{n m}+\sum_{p, q=0}^{d} V_{p q} x^{p} x^{q}\right. \\
& \left.-\sum_{p=0}^{d} x^{p}\left(V_{n p}+V_{m p}\right)\right) \quad(\mathrm{cf} . \text { N. Okada [7]) } \\
{\left[\alpha_{i} \cdot \alpha_{i}^{t}\right]^{n m}(x) } & =\sigma^{2} x^{n}\left(\delta_{n, m}-x^{m}\right)+x^{n} x^{m}\left(\sum_{r=0}^{d} \beta_{r} x^{r}-\beta_{n}-\chi_{m}\right) \\
& +\delta_{n, m} \beta_{n} x^{n} \quad \text { (cf. K. Sato [10]), }
\end{aligned}
$$

where $\sigma^{2} \geqq 0, x=\left\{x^{k}\right\}_{1 \leq k \leq d} \in L, x^{0}=1-\sum_{n=1}^{d} x^{n}, \quad\left\{V_{n m}\right\}_{0 \leq n, m \leq d}$ is nonnegative definite, $\beta_{n} \geqq 0 \quad(0 \leqq n \leqq d)$.

Now, we are in position to discuss the uniqueness of solutions of $E q$. (3.5). We will begin with the case $d=1$.

Assumption $[B-1]^{\prime} \quad \alpha_{i}(y), i \in S$ are real-valued $\frac{1}{2}$-Hölder continuous and uniformly bounded functions, which satisfy

$$
\alpha_{i}(0)=\alpha_{i}(1)=0 \text { for each } i \in S .
$$

From now on, the coordinate of a point $X \in[0,1]^{s}$ will be denoted by $X=\left\{x_{i}\right\}_{i \in S}$, where $x_{i} \in R^{1}$.

Assumption $[B-2]^{\prime} \quad f_{i}(X), i \in S$ are continuous functions defined on $[0,1]^{s}$ and satisfy the following conditions;

(3.13) there exists a matrix $Q=\left\{Q_{i, j}\right\}_{i, j \in s}$ such that $Q_{i, j} \geqq 0$ for all $i$ and $j, \sup _{i \in S} \sum_{j \in S} Q_{i, j}<+\infty$, and

$$
\left|f_{i}(X)-f_{i}(Y)\right| \leqq \sum_{j \in S} Q_{i, j}\left|x_{j}-y_{j}\right| \text { for any } X=\left\{x_{j}\right\}, Y=\left\{y_{j}\right\},
$$

(3. 14) $f_{i}(X) \geqq 0$ if $X=\left\{x_{j}\right\} \in[0,1]^{s}$ and $x_{i}=0$,

and 


$$
f_{i}(X) \leqq 0 \text { if } X=\left\{x_{j}\right\} \in[0,1]^{s} \text { and } x_{i}=1 .
$$

Then, Theorem 3.1 implies that under Assumptions $[B-1]^{\prime}$ and $[B-2]^{\prime}$ for any $X=\left\{x_{i}\right\} \in[0,1]^{s}$, the stochastic differential equation

$$
d x_{i}(t)=\alpha_{i}\left(x_{i}(t)\right) d B_{i}(t)+f_{i}(X(t)) d t \quad i \in S,
$$

where $\left\{B_{i}(t)\right\}_{i \in s}$ is an independent system of 1-dimensional Brownian motions, has a solution $\left\{(X(t), B(t)),\left(\Omega, \mathscr{F}, P ; \mathscr{F}_{t}\right)\right\}$ such that

$$
P\left[X(0)=X \text { and } X(t) \in[0,1]^{s} \text { for all } t \geqq 0\right]=1 .
$$

Furthermore, we obtain

Theorem 3.2. Let $X \in[0,1]^{s}$. Under Assumptions $[B-1]^{\prime}$ and $[B-2]^{\prime}$

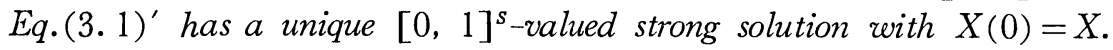

Proof. It is sufficient to show that the pathwise uniquencess of $E q$. (3. 1) $)^{\prime}$ holds. Let $(X(t), B(t))$ and $\left(X^{\prime}(t), B(t)\right)$ be two $[0,1]^{s}$-valued solutions of $(3.1)^{\prime}$ defined on $\left(\Omega, \mathscr{F}, P ; \mathscr{F}_{\imath}\right)$ with $X(0)=X^{\prime}(0)=X$. Choose a sequence of smooth functions on $R^{1},\left\{\psi_{p}(\xi)\right\}$ such that

$$
0 \leqq \psi_{p}(\xi) \nearrow|\xi| \text { as } p \rightarrow+\infty,\left|\psi_{p}^{\prime}(\xi)\right| \leqq 1 \text { and }\left\{|\xi| \psi_{p}^{\prime \prime}(\xi)\right\}_{p \geqq 1}
$$

is uniformly bounded and converges to 0 as $p \rightarrow+\infty$. Using Ito's formula for $\psi_{p}$ and letting $p \rightarrow+\infty$, we see that

$$
E\left[\left|x_{i}(t)-x_{i}^{\prime}(t)\right|\right] \leqq \sum_{j \in S} Q_{i, j} \int_{0}^{t} E\left[\left|x_{j}(s)-x_{j}^{\prime}(s)\right|\right] d s
$$

Noting that $E\left[\left|x_{i}(t)-x_{i}^{\prime}(t)\right|\right] \leqq 1$ and Assumption $[B-2]^{\prime}$, it follows evidently that $E\left[\left|x_{i}(t)-x_{i}^{\prime}(t)\right|\right]=0$ for any $t \geqq 0$ and $i \in S$. Thus, the proof is complete.

In this place, we can state precisely the example whose outline has already been mentioned at the beginning of $\S 1$.

Example. We put $\alpha_{i}(x)=\sqrt{\frac{1}{2 N} x(1-x)}$ for $x \in[0,1]$, and

$$
f_{i}(X)=v-(u+v) x_{i}+s x_{i}\left(1-x_{i}\right)+\sum_{j \in S} x_{j} q_{j i} \text { for } X=\left\{x_{j}\right\} \in\left[\begin{array}{ll}
0, & 1
\end{array}\right]^{s} .
$$

Here, $u$ and $v$ are non-negative constant, $s$ is a constant and we assume that $\left\{q_{j i}\right\}$ satisfies

$$
q_{j i} \geqq 0(i \neq j), \quad \sum_{j \in S} q_{j i}=0, \text { and } \sup _{j \in S}\left|q_{j j}\right|<+\infty .
$$


Then, since Assumptions $[B-1]^{\prime}$ and $[B-2]^{\prime}$ are fulfilled, Theorem 3.2 holds in this case.

Again, we will discuss the $d$-dimensional case. Our concern at this moment is to show the pathwise uniqueness of solutions of Eq.(3.1). However, it is rather difficult to show it without special conditions on the coefficients of (3.1) because the Lipschitz continuity of $\alpha_{i}^{n m}(x)$ cannot be assumed for our case.

Assumption $[B-1]^{\prime \prime} \quad \alpha_{i}(x)=\left\{\alpha_{i}^{n m}(x)\right\} \quad 1 \leqq n, m \leqq d$, are lower triangular matrices such that

$$
\alpha_{i} \cdot \alpha_{i}^{t}(x)=\left\{\sigma^{2} x^{n}\left(\delta_{n, m}-x^{m}\right)\right\}_{1 \leq n, m \leqq d, i \in S, x \in L}
$$

and $\alpha_{i}(x), i \in S$ are continuous on $L$.

Assumption $[B-2]^{\prime \prime}\left\{f_{i}^{n}(X)\right\}, i \in S, 1 \leq n \leq d$ satisfy Assumption [B-2]. Moreover $f_{i}^{n}(X)$ depends only on the first $n$-coordinates $\left\{\left(x_{i}^{1}, \ldots, x_{i}^{n}\right)\right\}_{i \in S}$ of $X=\left\{\left(x_{i}^{1}, \ldots, x_{i}^{d}\right)\right\}_{i \in s}$ for each $1 \leqq n \leqq d$ and $i \in S$, and

(3.17) there exists a matrix $Q=\left\{Q_{i, j}\right\}_{i, j \in s}$ such that $Q_{i, j} \geqq 0$ for all $i$ and $i, \sup _{i \in S} \sum_{j \in S} Q_{i, j}<+\infty$, and

$$
\left|f_{i}(X)-f_{i}(Y)\right| \leqq \sum_{j \in S} Q_{i, j}\left|x_{j}-y_{j}\right| \text { for any } X=\left\{x_{i}\right\}, \quad Y=\left\{y_{i}\right\} \in L^{s} .
$$

Here we note that $\alpha_{i}(x)$ of Assumption $[B-1]^{\prime \prime}$ is uniquely determined and satisfies that

(3.18) if $x^{k}=y^{k}$ for $k=1,2, \ldots, n-1$, then $\left|\alpha_{i}^{n m}(x)-\alpha_{i}^{n m}(y)\right| \leqq 2 \sigma \sqrt{\left|x^{n}-y^{n}\right|}$ for any $x$ and $y \in L$.

(cf. K. Sato [9])

Then we have

Theorem 3. 3. Under Assumption $[B-1]^{\prime \prime}$ and $[B-2]^{\prime \prime}$, the stochastic differentixl equation (3.1) has a unique $L^{s}$-valued strong solution with $X(0)=X$ for any $X \in L^{s}$.

Proof. The pathwise uniqueness of solutions of $E q .(3.1)$ can be proved by induction on $n$. First, we can see by Theorem 3.2 that $\left\{x_{i}^{1}(t)\right\}_{i \in S}$ is uniquely determined. Secondly, we assume that $\left\{x_{i}^{k}(t)\right\} i \in S$, $1 \leqq k \leqq n$ are uniquely determined. Then by (3.18) and the argument similar to the proof of Theorem 3.2, we can show that $\left\{x_{i}^{k+1}(t)\right\} \quad i \in S$ 
is uniquely determined. Thus, the proof is complete.

Remark 3.2. By Theorem 3.3 we can construct a strongly continuous Markov semi-group $\left\{T_{t}\right\}_{t \geq 0}$ on $C\left(L^{s}\right)$ such that

$$
T_{t} g-g=\int_{0}^{t} T_{s} A g d s \quad \text { for each } g \in C_{0}^{2}\left(L^{s}\right),
$$

where

$$
\begin{aligned}
& A g(X)=\sum_{i \in S}\left(\sum_{n, m=1}^{d} \sigma^{2} x_{i}^{n}\left(\delta_{n, m}-x_{i}^{m}\right) \frac{\partial^{2}}{\partial x_{i}^{n} \partial x_{i}^{m}} g(X)\right. \\
& \left.\quad+\sum_{n=1}^{d} \mathrm{f}_{i}^{n}\left(\left\{\left(x_{j}^{1}, x_{j}^{2}, \ldots, x_{j}^{n}\right)\right\}_{j \in S}\right)-\frac{\partial}{\partial x_{i}^{n}} g(X)\right),
\end{aligned}
$$

$C\left(L^{s}\right)$ denotes the set of all continuous functions on $L^{s}$ and $C_{0}^{2}\left(L^{s}\right)$ denotes the set of all $C^{2}$-functions on $L^{s}$ which depend only on finitely many coordinates.

Finally, we will discuss an infinite alleles model. Suppose that we have infinite number of alleles $A_{1}, A_{2}, \ldots, A_{n}, \ldots$ Denote by $x_{i}^{n}(t)$ the $A_{n}$-gene frequency in colony $i$ at time $t$. We expect that $\left\{\left(x_{i}^{1}(t), x_{i}^{2}(t)\right.\right.$, $\left.\left.\ldots, x_{i}^{n}(t), \ldots\right)\right\}_{i \in S}$ is a diffusion process on the state space $\left(L_{\infty}\right)^{\mathcal{S}}$ with its infinitesimal genetator

$$
A g(X)=\sum_{i \in S}\left(\sum_{n, m \in N} \sigma^{2} x_{i}^{n}\left(\delta_{n, m}-x_{i}^{m}\right) \frac{\partial^{2}}{\partial x_{i}^{n} \partial x_{i}^{m}} g(X)+\sum_{n \in N} f_{i}^{n}(X) \frac{\partial}{\partial x_{i}^{n}} g(X)\right)
$$

where $\boldsymbol{N}$ is the set of natural numbers.

Here, we restrict our treatment to the case that $f_{i}^{n}(X)$ can be expressed in the form

$$
f_{i}^{n}(X)=\sum_{k=1}^{n} \mu_{k n} x_{i}^{k}+\sum_{j \in S} q_{j i} x_{j}^{n} \text { for each } n .
$$

Suppose the following conditions on $\left\{\mu_{k n}\right\}$ and $\left\{q_{j i}\right\}$;

$$
\mu_{k n} \geqq 0 \quad(k \neq n), \quad \sum_{n=k}^{\infty} \mu_{k n}=0 \text { and } \sup _{n}\left|\mu_{n n}\right|<+\infty .
$$

and

$$
q_{j i} \geqq 0 \quad(i \neq j), \sum_{j \in S} q_{j i}=0 \text { and } \sup _{i \in S}\left|q_{i i}\right|<+\infty .
$$

In order to construct the above mentioned diffusion process, we consider the following stochastic differential equation

$$
d x_{i}^{n}(t)=\sum_{m=1}^{n} \alpha_{i}^{n m}\left(x_{i}^{1}(t), \ldots, x_{i}^{n}(t)\right) d B_{i}^{m}(t)+f_{i}^{n}(X(t)) d t \quad i \in S, n \in N
$$


where $B=\left\{\left(B_{i}^{1}(t), \ldots, B_{i}^{n}(t), \ldots\right)\right\}_{i \in S}$ is an independent system of infinite dimensional Brownian motions and $\left\{\alpha_{i}^{n m}\left(x^{1}, \ldots, x^{n}\right)\right\}_{1 \varliminf_{m} \varliminf_{n}}$ are continuous functions on $L_{n}=\left\{\left(x^{1}, \ldots, x^{n}\right) \in R^{n} ; x^{k} \geqq 0\right.$ for each $k$ and $\left.\sum_{k=1}^{n} x^{k} \leqq 1\right\}$ which satisfy

$$
\sum_{k=1}^{n \wedge m} \alpha_{i}^{n k}\left(x^{1}, \ldots, x^{n}\right) \alpha_{i}^{m k}\left(x^{1}, \ldots, x^{m}\right)=\sigma^{2} x^{n}\left(\delta_{n, m}-x^{m}\right)
$$

for any $n, m \in N$ and $x=\left(x^{1}, x^{2}, \ldots, x^{n}, \ldots\right) \in L_{\infty}$ Then we have

Theorem 3.4. Suppose that (3.20), (3.21), (3.22) and (3.24) hold. For any $X \in\left(L_{\infty}\right)^{s}$, the stochastic differential equation (3.23) has a unique $\left(L_{\infty}\right)^{s}$-valued strong solution with $X(0)=X$.

Proof. Note that all the assumptions of Theorem 3.3 are fulfilled when we restrict our observation to $\left\{\left(x_{i}^{1}, x_{i}^{2}, \ldots, x_{i}^{d}\right)\right\}_{i \in s}$. Hence the stochastic differential equation

$$
d x_{i}^{n}(t)=\sum_{m=1}^{n} \alpha_{i}^{n m}\left(x_{i}^{1}, x_{i}^{2}, \ldots, x_{i}^{n}\right) d B_{i}^{m}(t)+f_{i}(X(t)) d t \quad i \in S, 1 \leqq n \leqq d
$$

has a unique strong solution $X^{d}(t)=\left(x_{i}^{1}(t), \ldots, x_{i}^{d}(t)\right)$ with given initial data $\left(x^{1}, \ldots, x^{d}\right)$ and satisfies

$$
P\left[x_{i}^{n}(t) \geqq 0 \quad(1 \leqq n \leqq d), \text { and } \sum_{n=1}^{d} x_{i}^{n}(t) \leqq 1 \text { for all } t \geqq 0\right]=1 .
$$

Thus, Eq. (3.23) has a unique strong solution $X(t)=\left\{x_{i}^{n}(t)\right\}^{i} \in S_{, n \in N}$ satisfying $P\left[x_{i}^{n}(t) \geqq 0 \quad(n \in N)\right.$ and $\sum_{n=1}^{\infty} x_{i}^{n}(t) \leqq 1$ for all $\left.t \geqq 0\right]=1$.

To complete the proof it is sufficient to show that

$$
P\left[\sum_{n=1}^{\infty} x_{i}^{n}(t)=1 \text { for all } t \geqq 0 \text { and } i \in S\right]=1
$$

Set $Z_{i}(t)=1-\sum_{n=1}^{\infty} x_{i}^{n}(t)$. Then by (3.20) we can easily see

$$
\begin{aligned}
E\left[Z_{i}(t)\right] & =-\int_{0}^{t} \sum_{n=1}^{\infty} \sum_{k=1}^{n} \mu_{k n} E\left[x_{i}^{k}(s)\right] d s+\sum_{j \in S} q_{j i} \int_{0}^{t} E\left[Z_{j}(s)\right] d s \\
& =\sum_{j \in S} q_{j i} \int_{0}^{t} E\left[Z_{j}(s)\right] d s .
\end{aligned}
$$

Thus we have $E\left[Z_{i}(t)\right]=0$ for any $i \in S$ and $t \geqq 0$. Also, since we see easily that $Z_{i}(t)$ is a continuous function of $t \geqq 0$ almost surely, we obtain (3. 25). 


\section{§ 4. Diffusion processes on $\mathscr{S}^{\prime}\left(Z^{d}\right)$}

In this section we shall construct diffusion processes on $\mathscr{S}^{\prime}\left(Z^{d}\right)$ by means of stochastic differential equations. Also, we shall apply it to construct a time evolution of an unbounded spin system in statistical mechanics.

Let $Z^{d}$ be the $d$-dimensional Euclidean lattice space. $\mathscr{S}\left(Z^{d}\right)$ denotes the space of all rapidly decreasing (real) sequences on $Z^{d}$. We introduce the Hilbertian norms \|\|$_{p}, p \in Z^{1}$, on $R^{z^{d}}$;

$$
\|a\|_{p}^{2}=\sum_{i \in Z^{d}}(|i|+1)^{2 p}\left|a_{i}\right|^{2}, \quad a=\left\{a_{i}\right\}_{i \in Z^{d}} \in R^{z^{d}} .
$$

Then it is known that $\mathscr{S}\left(Z^{d}\right)$ is a nuclear space by the sequence of the norms $\left\{\|\|_{p} ; p=1,2, \ldots\right\}$.

Let us denote by $\mathscr{S}^{\prime}\left(Z^{d}\right)$ the dual space of $\mathscr{S}\left(Z^{d}\right)$. Then $\mathscr{S}^{\prime}\left(Z^{d}\right)$ coincides with the set of all tempered sequences on $Z^{d}$.

For each integer $p$, define $\mathscr{S}_{p}$ by

$$
\mathscr{S}_{p}\left(Z^{d}\right)=\left\{X=\left\{x_{i}\right\}_{i \in Z^{d}} \in R^{z^{d}} ;\|X\|_{p}<+\infty\right\} .
$$

Then, it holds that

$$
\mathscr{S}\left(Z^{d}\right)=\bigcap_{p} \mathscr{S}_{p}\left(Z^{d}\right) \text { and } \mathscr{S}^{\prime}\left(Z^{d}\right)=\cup_{p} \mathscr{S}^{p}\left(Z^{d}\right) .
$$

Here $\mathscr{S}^{\prime}\left(Z^{d}\right)$ is endowed with the strong topology. But we note that a curve $X_{t}, t \in[0, \infty)$ in $\mathscr{S}^{\prime}\left(Z^{d}\right)$ is strongly continuous if and only if it is weakly continuous. (cf. I. M. Gel' fand-N. Ya. Vilenkin [3])

Let us consider the following stochastic differential equation under the Assumptions [C-1] and [C-2].

$$
d x_{i}(t)=\alpha_{i}\left(x_{i}(t)\right) d B_{i}(t)+f_{i}\left(x_{i}(t), X(t)\right) d t \quad i \in Z^{d},
$$

where $X(t)=\left\{x_{i}(t)\right\}_{i \in Z^{d}}$ and $B(t)=\left\{B_{i}(t)\right\}_{i \in Z^{d}}$ is an independent system of one-dimensional Brownian motions on a probability space $\left(\Omega, \mathscr{F}, P ; \mathscr{F}_{t}\right)$.

Assumption [C-1] $\alpha_{i}, i \in Z^{d}$ are real continuous functions on $R^{1}$ which satisfy the following conditions;

$$
\sup _{i \in Z^{d}}\left|\alpha_{i}(0)\right|<+\infty
$$

and there exists a constant $L$ such that 


$$
\left|\alpha_{i}(x)-\alpha_{i}(y)\right| \leqq L|x-y|
$$

for any $x, y \in R^{1}$ and $i \in Z^{d}$.

Assumption [C-2] $f_{i}, i \in Z^{d}$ are real functions defined on $R^{1} \times \mathscr{S}^{\prime}\left(Z^{d}\right)$ which satisfy the following conditions;

(4.5) for any finite subset $V$ of $Z^{d}, f_{i}(x, X), i \in Z^{d}$ are locally

Lipschitz continuos in the variables $\left(x,\left\{x_{j}\right\}_{j \in V}\right)$, where $X=\left\{x_{j}\right\}_{j \in Z^{d}} \in \mathscr{S}^{\prime}\left(Z^{d}\right)$,

$$
\sup _{i \in z^{d}}\left|f_{i}(0,0)\right|<+\infty
$$

there exists a constant $K$ such that

$$
(x-y)\left(f_{i}(x, X)-f_{i}(y, X)\right) \leqq K(x-y)^{2} \text { for any } x, y \in R^{1}, X \in \mathscr{S}^{\prime}\left(Z^{d}\right)
$$
and $i \in Z^{d}$ and there exists a positive sequence $c=\left\{c_{i}\right\}_{i \in Z^{d}} \in \mathscr{S}\left(Z^{d}\right)$ such that

$$
\left(f_{i}(x, X)-f_{i}(x, Y)\right)^{2} \leqq \sum_{j \in Z^{d}} c_{i-j}\left(x_{j}-y_{j}\right)^{2}
$$

for any $X=\left\{x_{j}\right\}, Y=\left\{y_{j}\right\} \in \mathscr{S}^{\prime}\left(Z^{d}\right), x \in R^{1}$, and $i \in Z^{d}$.

Then, under Assumptions $[C-1]$ and $[C-2]$ we have

Theorem 4.1. For any $X \in \mathscr{S}^{\prime}\left(Z^{d}\right)$, the stochastic differential equation (4. 2) has a unique $\mathscr{S}^{\prime}\left(Z^{d}\right)$-valued strong solution $X(t)$ such that

$$
P[X(0)=X \text {, and } X(t) \text { is continuous in } t \geqq 0]=1 \text {. }
$$

For the proof we prepare some lemmas.

Lemma 4. 1. For $X=\left\{x_{j}\right\}, Y=\left\{y_{j}\right\} \in \mathscr{S}^{\prime}\left(Z^{d}\right)$ and $x, y \in R^{1}$,

$$
x f_{i}(x, X) \leqq\left(f_{i}(0,0)\right)^{2}+\left(K+\frac{1}{2}\right) x^{2}+\sum_{j \in z^{d}} c_{i-j} x_{j}^{2}
$$

and

$$
\operatorname{sgn}(x-y)\left(f_{i}(x, X)-f_{i}(y, Y)\right) \leqq\left(2 K^{2}(x-y)^{2}+2 \sum_{j \in Z^{d}} c_{i-j}\left(x_{j}-y_{j}\right)^{2}\right)^{1 / 2} .
$$

Proof. It is immediate from Assumption [C-2].

Lemma 4. 2. Let $c=\left\{c_{i}\right\}_{i \in Z^{d}}$ be a positive sequence of $\mathscr{S}\left(Z^{d}\right)$. Then there exists a positive sequence $\left\{K_{p}\right\}_{p \geq 1}$, a constant $K$ and a positive sequence $d=\left\{d_{i}\right\}_{i \in Z^{d}}$ 
of $\mathscr{S}\left(Z^{d}\right)$ such that

(i ) $\quad \sum_{i \in Z^{d}}(|i|+1)^{-2 p} c_{i-j} \leqq K_{p}(|j|+1)^{-2 p}$ for any $j \in Z^{d}$ and $p \in \boldsymbol{N}$

and

( ii)

$$
\sum_{i \in z^{d}} d_{i} c_{i-j} \leqq K d_{j} \text { for any } i \in Z^{d}
$$

Proof. We will prove only (ii) since (i) is easy.

Let $K>\sum_{i \in z^{d}} c_{i}$ and set $d_{i}=\sum_{n=0}^{\infty} \frac{\left(c^{* n}\right)^{i}}{K^{n}}$. Here $c^{* n}$ stands for the $n$-th convolution of $c=\left\{c_{i}\right\}$. Then the inequality of (ii) is obvious. Set

$$
\hat{d}(\theta)=\sum_{j \in z^{d}} e^{i<\theta, j>} d_{j} \text { and } \hat{c}(\theta)=\sum_{j \in Z^{d}} e^{i<\theta, j>} c_{j}
$$

where

$$
\langle\theta, j\rangle=\sum_{k=1}^{d} \theta_{k} j_{k} \text { for each } \theta=\left(\theta_{1} \ldots, \theta_{d}\right) \in R^{d}
$$

and $j=\left(j_{1}, \ldots, j_{d}\right) \in Z^{d}$. Then we see

$$
\hat{d}(\theta)=\frac{K}{K-\hat{c}(\theta)} .
$$

Since $\hat{c}(\theta)$ is a $C^{\infty}$-function and $.|\hat{c}(\theta)|<K$, it follows that $\hat{d}(\theta)$ is also a $C^{\infty}$-function. Thus, $d=\left\{d_{i}\right\} \in \mathscr{S}\left(Z^{d}\right)$ follows from this (cf. Loève [5], p. 199)

Lemma 4. 3. Let $\gamma=\left\{\gamma_{i}\right\}_{i \in Z^{d}}$ and $a=\left\{a_{i}\right\}_{i \in Z^{d}}$ be non-negative sequences on $Z^{d}$ and let $Q=\left\{Q_{i, j}\right\}_{i, j \in Z^{d}}$ be a non-negative matrix on $Z^{d} \times Z^{d}$. Let $\left\{u_{i}(t)\right\}_{i \in Z^{d}}$ be a sequence of non-negative measurable functions defined on a finite interval $[0, T]$.

Suppose that the following condition are satisfied;

$$
\begin{gathered}
\sum_{i \in Z^{d}} \gamma_{i} a_{i}<+\infty, \\
\sum_{i \in Z^{d}} \gamma_{i} Q_{i, j} \leqq K \gamma_{j}, j \in Z^{d}, \text { hold for some } K>0, \\
\sum_{i \in Z^{d}} \gamma_{i} \sup _{0 \leq i \leq T} u_{i}(t)<+\infty,
\end{gathered}
$$

and

$$
u_{i}(t) \leqq a_{i}+\sum_{j \in Z^{d}} Q_{i, j} \int_{0}^{t} u_{j}(s) d s, \quad i \in Z^{d}
$$

Then it holds that 
(4.. 14) $u_{i}(t) \leqq \sum_{j \in z^{d}}(\exp \{t Q\})_{i, j} a_{j}$ for any $i \in Z^{d}$ and $t \in[0, T]$

and

$$
\sum_{i \in Z^{d}} \gamma_{i} \sup _{0 \leq t \leq T} u_{i}(t) \leqq e^{T K} \sum_{j \in Z^{d}} \gamma_{i} a_{i}
$$

Proof. It is obvious.

Proof of Theorem 4. 1.

Let $X \in \mathscr{S}^{\prime}\left(Z^{d}\right)$. Then $X \in \mathscr{S}_{-p}$ for some $p \geqq d$. Let $\left\{S_{n}\right\}$ be a sequence of finite subsets of $Z^{d}$ such as $S_{n} \nearrow S(n \rightarrow \infty)$.

Consider the following stochastic differential equations;

$$
\begin{aligned}
& x_{i}^{n}(t)=x_{i} \quad i \in S_{n} \\
& x_{i}^{n}(t)=x_{i}+\int_{0}^{t} \alpha_{i}\left(x_{i}^{n}(s)\right) d B_{i}(s)+\int_{0}^{t} f_{i}\left(x_{i}^{n}(s), X^{n}(s)\right) d s, \quad i \in S_{n},
\end{aligned}
$$

where $X^{n}(t)=\left\{x_{j}^{n}(t)\right\}_{j \in Z^{d}}$.

Then it is easy to see that $E q .(4.16)$ is uniquely solvable and satisfies (4. 17) $\sup _{0 \leq t \leq T} E\left[\left|x_{i}^{n}(t)\right|^{2}\right]<+\infty$ for any finite $T>0$ and $i \in Z^{d}$.

First, we claim that

(4. 18) $\sum_{i \in z^{d}}(|i|+1)^{-2 p} \sup _{n} E\left[\sup _{0 \leq t \leq T}\left|x_{i}^{n}(t)\right|^{2}\right]<+\infty$ for any finite $T>0$.

For simplicity, we will show (4.18) under an additional assumption;

$$
\sup _{i \in Z^{d}}\left\|\alpha_{i}\right\|<+\infty, \text { where }\left\|\alpha_{i}\right\|=\sup _{y \in R^{1}}\left|\alpha_{i}(y)\right| .
$$

By Ito's formula, we see

$$
\begin{aligned}
\left(x_{i}^{n}(t)\right)^{2} & =x_{i}^{2}+2 \int_{0}^{t} x_{i}^{n}(s) \alpha_{i}\left(x_{i}^{n}(s)\right) d B_{i}(s)+\int_{0}^{t}\left(\alpha_{i}\left(x_{i}^{n}(s)\right)^{2}\right. \\
& \left.+2 x_{i}^{n}(s) f_{i}\left(x_{i}^{n}(s), X^{n}(s)\right)\right) d s, \quad i \in S_{n} .
\end{aligned}
$$

Also, it follows from a maximal inequality for martingales and Lemma 4. 1 that

$$
\begin{aligned}
& E\left[\sup _{0 \leq u \leq t}\left(x_{i}^{n}(u)\right)^{2}\right] \leqq x_{i}^{2} \\
&+4\left(\int_{0}^{t} E\left[\left(x_{i}^{n}(s) \alpha_{i}\left(x_{i}^{n}(s)\right)\right)^{2}\right] d s\right)^{1 / 2}+\int_{0}^{t} E\left[\alpha_{i}\left(x_{i}^{n}(s)\right)^{2}\right. \\
&\left.+2 f_{i}(0,0)^{2}+(2 K+1) x_{i}^{n}(s)^{2}+2 \sum_{j \in z^{d}} c_{i-j} x_{j}^{n}(s)^{2}\right] d s
\end{aligned}
$$

Thus, we have

(4. 20) $E\left[\sup _{0 \leq u \leq t}\left(x_{i}^{n}(u)\right)^{2}\right] \leqq z_{i}+\sum_{j \in z^{d}} Q_{i j} \int_{0}^{t} E\left[x_{j}^{n}(s)^{2}\right] d s, \quad i \in Z^{d}, t<T$, 
where $z_{i}=x_{i}^{2}+2\left\|\alpha_{i}\right\|+\left(\left\|\alpha_{i}\right\|^{2}+2 f_{i}(0,0)^{2}\right) T$ and

$$
Q_{i, j}= \begin{cases}2 c_{i-j} & (i \neq j) \\ 2 c_{0}+2\left\|\alpha_{i}\right\|+2 K+1 & (i=j)\end{cases}
$$

Set

$$
u_{i}(t)=E\left[\sup _{0 \leq u \leq t}\left(x_{i}^{n}(u)\right)^{2}\right], a_{i}=z_{i} \text { and } \gamma_{i}=(|i|+1)^{-2 p} .
$$

Then, noting Lemma 4. 2, we see that Lemma 4.3 is applicable for (4.20) Hence we obtain (4.18).

Secondly, we claim that

$$
\lim _{n, m \rightarrow \infty} E\left[\sup _{0 \leq t \leq T}\left\|X^{n}(t)-X^{m}(t)\right\|_{-p}^{2}\right]=0
$$

for any finite $T>0$. Let $m>n$ and $i \in S_{n}$. Then,

$$
\begin{aligned}
x_{i}^{m}(t)-x_{i}^{n}(t) & =\int_{0}^{t}\left(\alpha_{i}\left(x_{i}^{m}(s)\right)-\alpha_{i}\left(x_{i}^{n}(s)\right) d B_{i}(s)\right. \\
& +\int_{0}^{t}\left(f_{i}\left(x_{i}^{m}(s), X^{m}(s)\right)-f_{i}\left(x_{i}^{n}(s), X^{n}(s)\right) d s .\right.
\end{aligned}
$$

Applying Ito's formula for $\left\{\psi_{k}\right\}$ as in the proof of Theorem 3.2, we get

$$
\begin{aligned}
& \left|x_{i}^{m}(t)-x_{i}^{n}(t)\right| \\
& \quad=\int_{0}^{t} \operatorname{sgn}\left(x_{i}^{m}(s)-x_{i}^{n}(s)\right)\left(\alpha_{i}\left(x_{i}^{m}(s)\right)-\alpha_{i}\left(x_{i}^{n}(s)\right) d B_{i}(s)\right. \\
& \quad+\int_{0}^{t} \operatorname{sgn}\left(x_{i}^{m}(s)-x_{i}^{n}(s)\right)\left(f_{i}\left(x_{i}^{m}(s), X^{m}(s)\right)-f_{i}\left(x_{i}^{n}(s), X^{n}(s)\right)\right) d s .
\end{aligned}
$$

By using Lemma 4.1 and a maximal inequality for martingales,

$$
\begin{aligned}
& E\left[\sup _{0 \leq u \leq t}\left(x_{i}^{m}(u)-x_{i}^{n}(u)\right)^{2}\right] \leqq 8 \int_{0}^{t} E\left[\left(\alpha_{i}\left(x_{i}^{m}(s)\right)-\alpha_{i}\left(x_{i}^{n}(s)\right)^{2}\right)\right] d s \\
& \quad+4 T \int_{0}^{t} E\left[K^{2}\left(x_{i}^{m}(s)-x_{i}^{n}(s)\right)^{2}+\sum_{j \in Z^{d}} c_{i-j}\left(x_{j}^{m}(s)-x_{j}^{n}(s)\right)^{2}\right] d s .
\end{aligned}
$$

Setting $E\left[\sup _{0 \leq u \leq t}\left(x_{i}^{m}(u)-x_{i}^{n}(u)\right)^{2}\right]=N_{i}^{n, m}(t)$, we see

(4.23) $N_{i}^{n, m}(t) \leqq \sup _{n} E\left[\sup _{0 \leq i \leq T}\left(x_{i}^{n}(t)\right)^{2}\right] \cdot I_{S_{m} \backslash s_{n}}(i)+\sum_{j \in Z^{d}} Q_{i, j} \int_{0}^{t} N_{j}^{n, m}(s) d s$,

for any $i \in Z^{d}$ and $t \in[0, T]$, where

$$
I_{A}(i)=\left\{\begin{array}{ll}
1 & (i \in A) \\
0 & (i \notin A)
\end{array} \text { and } Q_{i, j}=\left\{\begin{array}{ll}
4 T c_{i-j} & (i \neq j) \\
8 L^{2}+4 T\left(K^{2}+c_{0}\right) & (i=j)
\end{array} .\right.\right.
$$

Applying Lemma 4.3 for $u_{i}(t)=N_{i, m}^{n, m}(t), Q=\left\{Q_{i, j}\right\}$ and $\gamma_{i}=(|i|+1)^{-2 p}$, we obtain 
(4. 24) $\sum_{i \in Z^{d}}(|i|+1)^{-2 p} N_{i}^{n, m}(T) \leqq e^{T\|Q\|} \sum_{j \in S_{m} \backslash s_{n}}(|j|+1)^{-2 p} M_{j}(T)$,

where

$$
M_{j}(T)=\sup _{n} E\left[\sup _{0 \leq i \leq T}\left(x_{j}^{n}(t)\right)^{2}\right]
$$

and

$$
\|Q\|=\sup _{j \in Z^{d}}\left(\sum_{i \in Z^{d}}\left(\frac{|j|+1}{|i|+1}\right)^{2 p} Q_{i, j}\right)
$$

Thus noting (4.18) we get (4.21).

Thirdly, it follows from (4.21) that there exists a $\mathscr{S}_{-p}$-valued strongly continuous process $\{X(t)\}_{t \geq_{0}}$ such that

$$
\lim _{n \rightarrow \infty} E\left[\sup _{0 \leq 1 \leq T}\left\|X^{n}(t)-X(t)\right\|_{-p}^{2}\right]=0 \text { for any finite } T>0 .
$$

Hence for suitable subsequence $\left\{n_{k}\right\}$,

$$
P\left[\lim _{k \rightarrow \infty} \sup _{0 \leq t \leq T}\left\|X^{n} k(t)-X(t)\right\|_{-p}=0 \text { for any finite } T>0\right]=1 .
$$

Accordingly, it follows easily that $(X(t), B(t))$ is a $\mathscr{S}^{\prime}\left(Z^{d}\right)$-valued solution of Eq.(4.2) and $X(t)$ is continuous in $t \geqq 0$ in the strong topology of $\mathscr{S}^{\prime}\left(Z^{d}\right)$ almost surely.

Finally, we will show the uniqueness of solutions. Suppose that $(X(t)$, $B(t))$ and $(Y(t), B(t))$ are two $\mathscr{S}^{\prime}\left(Z^{d}\right)$-valued solutions of $E q .(4.2)$ such that

$P[X(0)=Y(0)=X$ and $X(t)$ and $Y(t)$ are continuous in $t \geqq 0]=1$. By Lemma 4.2 there exists a positive sequence $d=\left\{d_{i}\right\}_{i \in Z^{d}}$ of $\mathscr{S}\left(Z^{d}\right)$ and a constant $K$ such that

$$
\text { (4. 25) } \sum_{i \in z^{d}} d_{i} c_{i-j} \leqq K d_{j} \text { for any } j \in Z^{d} \text {. }
$$

For each $n \in N$, define

$$
U_{n}=\left\{X \in \mathscr{S}^{\prime}\left(Z^{d}\right) ; \sup _{i \in Z^{d}}\left|x_{i}\left(d_{i}\right)^{1 / 4}\right|<n\right\} .
$$

Then it is easy to see that $U_{n}$ is a open subset of $\mathscr{S}^{\prime}\left(Z^{d}\right)$ in the strong topology. Let us introduce a sequence of stopping times $\left\{\tau_{n}\right\}$,

$$
\tau_{n}=\inf \left\{t \geqq 0 ; X(t)-X \notin U_{n} \text { or } Y(t)-X \notin U_{n}\right\} .
$$

By the continuity of $X(t)$ and $Y(t)$, we have

$$
P\left[\tau_{*}>0\right]=1 \text {. }
$$

Also, 


$$
\begin{aligned}
& E\left[\left(y_{i}\left(t \wedge \tau_{n}\right)-x_{i}\left(t \wedge \tau_{n}\right)\right)^{2}\right]=E\left[\int_{0}^{t \wedge \tau_{n}}\left(\alpha_{i}\left(y_{i}(s)-\alpha_{i}\left(x_{i}(s)\right)\right)^{2} d s\right]\right. \\
& \quad+E\left[\int_{0}^{t \wedge \tau_{n}}\left(y_{i}(s)-x_{i}(s)\right)\left(f_{i}\left(y_{i}(s), Y(s)\right)-f_{i}\left(x_{i}(s), X(s)\right)\right) d s\right] \\
& \quad \leqq \sum_{j \in Z^{d}} Q_{i, j} \int_{0}^{t} E\left[\left(y_{j}\left(s \wedge \tau_{n}\right)-x_{j}\left(s \wedge \tau_{n}\right)\right)^{2}\right] d s,
\end{aligned}
$$

where

$$
Q_{i, j}=\left\{\begin{array}{lc}
c_{i-j} & (i \neq j) \\
L^{2}+K+1+c_{0} & (i=j)
\end{array} .\right.
$$

Set $u_{i}(t)=E\left[\left(y_{i}\left(t \wedge \tau_{n}\right)-x_{i}\left(t \wedge \tau_{n}\right)\right)^{2}\right]$ and $\gamma_{i}=d_{i}$ for each $i \in Z^{d}$.

Then we see that Lemma 4.3 is applicable for (4.27).

Hence, $E\left[\left(y_{i}\left(t \wedge \tau_{n}\right)-x_{i}\left(t \wedge \tau_{n}\right)\right)^{2}\right]=0$ for any $n \in N, i \in Z^{d}$ and $t>0$ Since this implies $P[X(t)=Y(t)$ for all $t]=1$, we complete the proof of Theorem 4. 1.

Next, we shall apply Theorem 4.1 to construct a time evolution of an unbounded spin system.

Let $\Phi_{i}(x), i \in Z^{d}$ be $C^{2}$-functions defined on $R^{1}$ and let $\Phi_{i, j}(x, y), i, j \in Z^{d}$ be symmetric $C^{2}$-functions defined on $R^{2}$. Let us consider the potentials of the following type.

For each subset $V$ of $Z^{d}$

$$
U_{V}(X)=\sum_{i \in V} \Phi_{i}\left(x_{i}\right)+\frac{1}{2} \sum_{i+j \in V} \Phi_{i, j}\left(x_{i}, x_{j}\right)+\sum_{i \in V, k \notin V} \Phi_{i, k}\left(x_{i}, x_{k}\right),
$$

where $X=\left\{x_{j}\right\}_{j \in z^{d}} \in R^{z^{d}}$.

A time evolution associated with the potential functions $\left\{U_{v}\right\}_{v \subset z^{d}}$ is defined by the following stochastic differential equation;

(4. 29) $d x_{i}(t)=d B_{i}(t)-\frac{1}{2}\left(\Phi_{i}^{\prime}\left(x_{i}(t)\right)+\sum_{j \neq i} \Phi_{i, j}^{\prime}\left(x_{i}(t), x_{j}(t)\right)\right) d t, \quad i \in Z^{d}$,

where $B(t)=\left\{B_{i}(t)\right\}$ is an independent system of 1-dimensional Brownian motions, $\Phi_{i}^{\prime}(x)=\frac{d}{d x} \Phi_{i}(x)$ and $\Phi_{i, j}^{\prime}(x, y)=\frac{\partial}{\partial x} \Phi_{i, j}(x, y)$.

Assumption [C]' There exist a constant $K>0$, a positive matrix $Q=$ $\left\{Q_{i, j}\right\}_{i, j \in Z^{d}}$, satisfying $\sup _{i \in Z^{d}} \sum_{j \neq i} Q_{i, j}<+\infty$, and a positive sequence $c=\left\{c_{i}\right\}_{i \in z^{d}}$ of $\mathscr{S}\left(Z^{d}\right)$ such that

(4.30) $\sup _{i \in z^{d}}\left|\Phi_{i}^{\prime}(0)\right|<+\infty$ and $\Phi^{\prime \prime}(x) \geqq-K$, 
(4.31) $\sup _{i \in Z^{d}} \sum_{j \neq i}\left|\Phi_{i, j}^{\prime}(0,0)\right|<+\infty$ and $\sum_{j \not>i} \frac{\partial}{\partial x^{2}} \Phi_{i, j}\left(x, y_{j}\right)$ is uniformly convergent in $x$ on each bounded interval for any $X=\left\{x_{i}\right\} \in \mathscr{S}^{\prime}(Z)$ and

$$
\frac{\partial^{2}}{\partial x^{2}} \Phi_{i, j}(x, y) \geqq-Q_{i, j}, \text { and }\left|\frac{\partial^{2}}{\partial x \partial y} \Phi_{i, j}(x, y)\right| \leqq c_{i-j}
$$

for any $x, y \in R^{1}$ and $i, j \in Z^{d}$.

Then, under Assumption $[C]^{\prime}$ we have

Theorem 4. 2. Let $X \in \mathscr{S}^{\prime}\left(Z^{d}\right)$. Then the stochastic differentialequation (4.29) has a unique $\mathscr{S}^{\prime}\left(Z^{d}\right)$-valued strong solution $X(t)$ such that $P[X(0)=X$ and $X(t)$ is continuous in $t \geqq 0]=1$.

\section{Proof. Let}

$$
f_{i}(x, X)=-\frac{1}{2}\left(\Phi_{i}^{\prime}(x)+\sum_{j \neq i} \Phi_{i, j}^{\prime}\left(x, x_{j}\right)\right) \text { for } X=\left\{x_{j}\right\} \in \mathscr{S}^{\prime}\left(Z^{d}\right) .
$$

Then we can easily see that $\left\{\alpha_{i}(x) \equiv 1, f\left(x, X_{i}\right)\right\}$ satisfies Assumption [C-1] and $[C-2]$. Hence it follows from Theorem 4. 1.

Remark 4. 1. Denote by $X(t: X)$ the solution of Eq.(4.29) with the initial condition $X(0)=X$. Then we can define a $\mathscr{S}^{\prime}\left(Z^{d}\right)$-valued diffusion process $\left(X(t), P_{X}\right)_{X \in \mathscr{P}^{\prime}\left(Z^{d}\right)}$ by $E_{X}[f(X(t))]=E(f(X(t: X))]$ for every bounded measurable function $f$ on $\mathscr{S}^{\prime}\left(Z^{d}\right)$.

Let us denote by $\mathscr{P}\left(\mathscr{S}^{\prime}\left(Z^{d}\right)\right)$ the set of all probability measures on $\mathscr{S}^{\prime}\left(Z^{d}\right) \cdot \mu \in \mathscr{P}\left(\mathscr{S}^{\prime}\left(Z^{d}\right)\right)$ is called a reversible stationary state of $(X(t)$, $\left.P_{X}\right)_{x \in \mathscr{S}^{\prime}\left(Z^{d}\right)}$ if it satisfies the following condition;

$$
\int \mu(d X) E_{X}[f(X(t))] g(X)=\int \mu(d X) E_{X}[g(X(t))] f(X)
$$

hold for all bounded measurable functions $f$ and $g$ on $\mathscr{S}^{\prime}\left(Z_{d}\right)$.

From now on, we shall suppose Assumption $[C]^{\prime}$ and the following; (4.34) there exist some constant $A$ satisfying $A>\sum_{i \in Z^{d}} c_{j}$ and a constant $B$ such that

$$
x \Phi_{i}^{\prime}(x) \geqq A x^{2}-B \text { for any } x \in R^{1} \text {, and } i \in Z^{d} .
$$


Then, we can define Gibbs states associated with the potential functions $\left\{U_{v}\right\}_{v \subset z^{d}}$ of (4.28), (cf. G. Royer [8]). In particular, we denote by $\mathscr{G}_{T}(\Phi)$ the set of all Gibbs states associated with $\left\{U_{V}\right\}_{V \subset z^{d}}$ which are supported by $\mathscr{S}^{\prime}\left(Z^{d}\right)$.

\section{Theorem 4. 3 .}

( i ) $\mathscr{G}_{T}(\Phi)$ is not empty,

and

(ii) $\mu \in \mathscr{P}\left(\mathscr{S}^{\prime}\left(Z^{d}\right)\right)$ is a reversible stationary state of $\left(X(t), P_{X}\right)_{X \in \mathscr{I}^{\prime}\left(Z^{d}\right)}$, if and only if $\mu \in \mathscr{G}_{T}(\Phi)$.

Proof. This theorem is essentially due to H. Doss and G. Royer [2]. Since the reader can consult [2], we will omit the details of the proof.

DePARTMENT OF MATHEMATics

NARA WOMEN'S UNIVERSJTY

DEPARTMENT OF MATHEMATICS

NAGOYA INSTITUTE OF TECHNOLOGY

\section{References}

[1] J. F. Crow and M. Kimura, An introduction to population genetics theory, Harper Row, (1970).

[2] H. Doss et G. Royer, Processus de diffusion associe aux mesures de Gibbs sur $R^{Z^{d}}, Z$. W. 46, 107-124, (1978).

[3] I. M. Gel' fand and N. Ya. Vilenkin, Generalized functions IV, Academic Press, (1964).

[4] N. V. Krylov, On Ito's stochastic differential equations, Theory of prob. and its appl., 14, 330-336, (1969).

[5] M. Loéve, Probability theory, Van Nostrand Reinhold Company, (1963).

[6] T. Maruyama, Stochastic problems in population genetics, Lecture notes in biomathematics, 17, Springer-Verlag, (1977).

[7] N. Okada, On convergence to diffusion processes of Markov chains related to population genetics, Adv. Appl. Prob. 11, 673-700, (1979).

[8] G. Royer, Etude de champs euclidiens sur un reseau on $Z^{\nu}$, J. Math. pure et appl., 56, 455-478, (1977).

[ 9 ] K. Sato, Diffusion processes and a class of Markov chains related to population genetics, Osaka J. Math., 13, 631-659, (1976).

[10] K. Sato, Convergence to a diffusion of a multi-allelic model in population genetics. Adv. Appl. Prob., 10, 538-562, (1978).

[11] T. Shiga, An interacting system in population genetics, J. Math. Kyoto Univ., 20-2, 213-242, (1980).

[12] A. V. Skorohod, Studies in the theory of random processes, Kiev, (1961), (English translation, Addison Wesley, 1965).

[13] T. Yamada and S. Watanabe, On the uniqueness of solutions of stochastic differential equations, J. Math. Kyoto Univ., 11, 155-167, (1971).

[14] S. Watanabe, Stochastic differential equations (in Japanese) Sangyo-Tosho, (1975). 